\title{
Distribution of Calcium Currents in Sprouting PC12 Cells
}

\author{
Jürg Streit and Hans Dieter Lux \\ Max-Planck-Institute for Psychiatry, Department of Neurophysiology, D-8033 Planegg-Martinsried, Federal Republic of \\ Germany
}

\begin{abstract}
Whole-cell calcium and barium currents were recorded from PC12 cell bodies and growth cones during nerve growth factor (NGF)-induced neurite outgrowth. Depolarizing voltage steps were applied to activate the currents, and pharmacological agents were used to separate them from other ionic currents. In recordings from growth cones still attached to neurites, current flow from central parts of the cell could be distinguished from the growth cone currents. On the other hand, currents from neuritic shafts and growth cones contributed to whole-cell currents recorded in the soma. Such currents were isolated by alternatively comparing recordings of whole-cell calcium currents with recordings in which calcium currents of defined parts of the cell were suppressed by exposing these regions to laminar streams of solutions with low calcium. The boundary between such streams and the bath solution was shown to be sharp using a calciumselective microelectrode. The current deficits recorded when the growth cones were exposed to solutions with low calcium (growth cone currents) were within 10-50\% of the total cell currents and similar to the currents recorded when the whole cell except the growth cone was exposed to low calcium. The current densities In the growth cones during initial sprouting were 5.4 times higher than those in the somata. Growth cone currents showed more inactivation than currents originating from the soma during pulses of 200 msec. In most experiments no calcium currents could be resolved in the neuritic shaft during initial neurite growth (1-10 d of NGF application), indicating low current density. In proximal segments of the neurites, however, a somatofugal decrease of the current density was observed. It is concluded from these results, that in outgrowing neurites of PC12 cells high densities of calcium channels are maintained in the growth cones, whereas in the neuritic shaft calcium channel density is initially low and later increases during consolidation of the neurites.
\end{abstract}

Among the variety of cellular functions put forward for the calcium channels a role in the regulation of neurite growth has also been proposed (Llinás, 1979; Anglister et al., 1982; Freeman et al., 1985; Mattson and Kater, 1987), an argument that implies that calcium channels are distributed over the whole neuron, including the processes. Evidence for the existence of calcium channels in neurites is based on the analysis of action potentials (Llinás and Sugimori, 1980; Llinás and Yarom, 1981),

\footnotetext{
Received Nov. 15, 1988; revised Apr. 21, 1989; accepted June 5, 1989.

We would like to thank Drs. 1. Dietzel-Meyer and J. McGuigan for the discussion of the manuscript and V. Bulka for the excellent technical assistance.

Correspondence should be addressed to Jürg Streit, Physiologisches Institut der Universität, Bühlplatz 5, CH-3012 Bern, Switzerland.

Copyright (C) 1989 Society for Neuroscience $0270-6474 / 89 / 124190-10 \$ 02.00 / 0$
}

notch currents (Yaari et al., 1987), or single-channel recordings under patch-clamp conditions (Bley et al., 1987; Niesen et al., 1987), experiments in which a comparison of current densities between somatic and dendritic parts of the neuron was not possible. A region of special interest for the hypothesis of growth regulation by calcium channels is the frontier zone of the neurite, the growth cone. Several authors reported electrical excitability (Willard, 1980), action potentials (Meiri et al., 1981), extracellular signals (Anglister et al., 1982; Freeman et al., 1985), and optical signals (Grinvald and Farber, 1981; Bolsover and Spector, 1986; Connor, 1986; Cohan et al., 1987; Lipscombe et al., 1988) from growth cone areas of various cells, indicating the existence of voltage-dependent calcium channels in these structures. These findings are strongly supported by direct whole-cell (Streit and Lux, 1986, 1987; Marom and Dagan, 1987) and single-channel (Lipscombe et al., 1988) current recordings under voltage-clamp conditions. However, they could not be confirmed in all preparations (Belardetti et al., 1986; Ross et al., 1988a, but see also Ross et al., 1988b).

In a previous paper (Streit and Lux, 1987), we reported the existence of voltage-dependent calcium currents in mechanically isolated growth cones of PC12 cells. Regulation of sprouting of these cells by nerve growth factor (NGF) facilitates the study of the relationships between cell growth and the density and distribution of calcium channels. Comparison between the current densities in the somata and growth cones revealed relatively high constant values for the growth cones versus initially small and later increasing values for somata. However, for methodical reasons we were not able to record growth cone currents in the initial phase of neurite outgrowth, and comparison of current densities in later phases were based on values from different groups of cells. In addition, the neuritic shaft could not be directly studied.

In the present paper we compare current densities in the cell body, the neurites, and growth cones of individual cells during initial sprouting. For this, the contributions of calcium currents from growth cones and neuritic shafts to the whole-cell currents were measured by comparing whole-cell currents with recordings, where calcium currents were suppressed in defined parts of the cell by exposing these regions to a laminar flow of a low calcium solution. The error induced by space-clamp inhomogeneities was kept minimal by limiting this method to cells with processes of short electrotonic length.

A short report about preliminary results has already been published (Streit and Lux, 1988).

\section{Materials and Methods}

Cell culture. PC12 cells are a tumor cell line derived from rat pheochromocytoma (for review, see Greene and Tischler, 1982). Their cultivation followed standard methods (see also Streit and Lux, 1987). The 

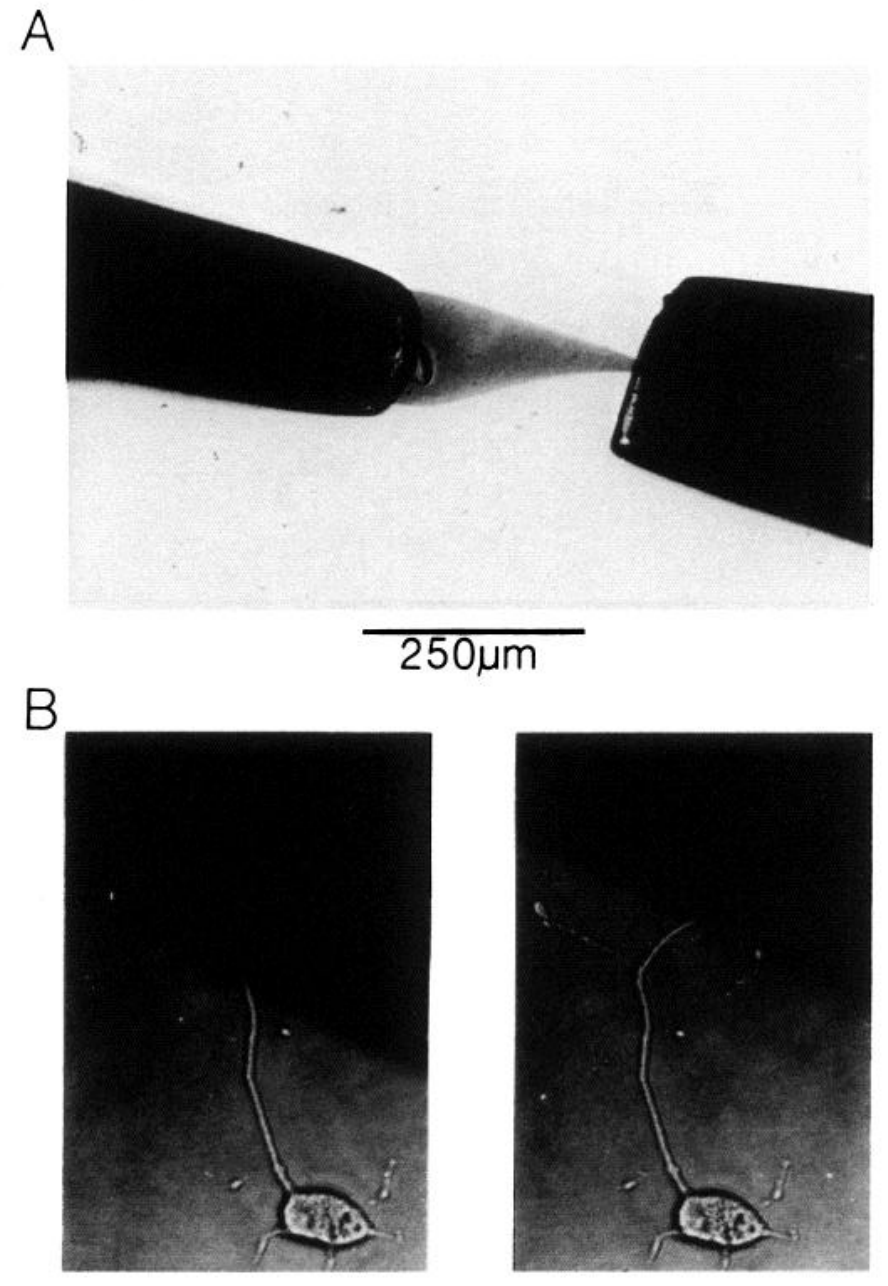

$100 \mu \mathrm{m}$

Figure 1. Laminar flow system for superfusion of a circumscribed part of the cell with test solution. $A$, Tips of the inflow (left) and the outflow (right) pipette. The test solution stream is stained by Fast green. $B$, Two distal segments of a sprouting PC12 cell exposed to test solution (dark).

cells were kept frozen at passage 6 and, when defrozen, grown in culture flasks up to passage 15 . The subculturing procedure was done every $7-$ $10 \mathrm{~d}$ and involved dissolution of the cells from the culture flask by $0.05 \%$ trypsin, centrifugation at low speed $(1200 \mathrm{rpm})$, and recultivation at 4 times lower density in a new culture flask or, for experimental use, in polyornithine-coated plastic culture dishes (Nunclon, Delta, Danmark). The cells were grown in modified Eagles medium containing 2 $\mathrm{mg} / \mathrm{ml} \mathrm{NaHCO}, 10 \%$ heat-inactivated horse serum, and $5 \%$ heat-inactivated fetal calf serum in an incubator at $37^{\circ} \mathrm{C}$ and a $\mathrm{CO}_{2}$ concentration of $5 \%$. 2.5S-NGF, if used, was added at a concentration of 0.5 $\mu \mathrm{g} / \mathrm{ml}$.

Solutions. The following external solutions were used in the bath and laminar flow system (mM):

1. $\mathrm{NaCl}, 120 ; \mathrm{CaCl}_{2}, 10 ; \mathrm{MgCl}_{2}, 1$; HEPES, 10; glucose, 10 .

2. $\mathrm{NaCl}, 140 ; \mathrm{CaCl}_{2}, 0 ; \mathrm{MgCl}_{2}, 1$; HEPES, 10 ; glucose, 10 .

3. $\mathrm{NaCl}, 60 ; \mathrm{BaCl}_{2}, 50 ; \mathrm{MgCl}_{2}, 1 ;$ HEPES, 10 ; glucose, 10 .

The $\mathrm{pH}$ was adjusted to 7.35 using $\mathrm{NaOH}$ in all solutions. $3 \mu \mathrm{M}$ TTX was added to all solutions to block TTX-sensitive sodium channels. The pipette solution contained (mM): $N$-methylglucamine- $\mathrm{Cl}, 100$; TEA$\mathrm{Cl}, 20$; EGTA, $10 ; \mathrm{MgCl}_{2}, 2$; HEPES, 10; glucose, 10; ATP, 2; $\mathrm{pH}=$ 7.35 .

Experimental set-up. For the experiments the culture medium in the dishes was replaced by one of the external solutions. Experiments were performed at room temperature $\left(22^{\circ} \mathrm{C}\right)$. The measurements of the di-

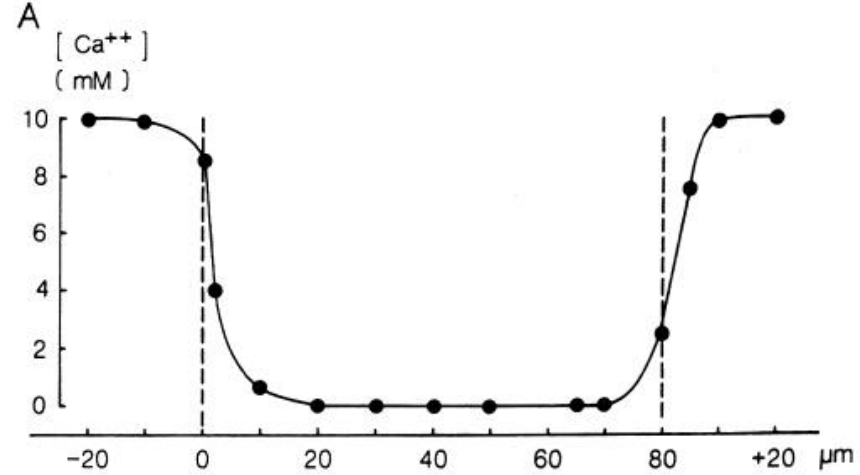

B
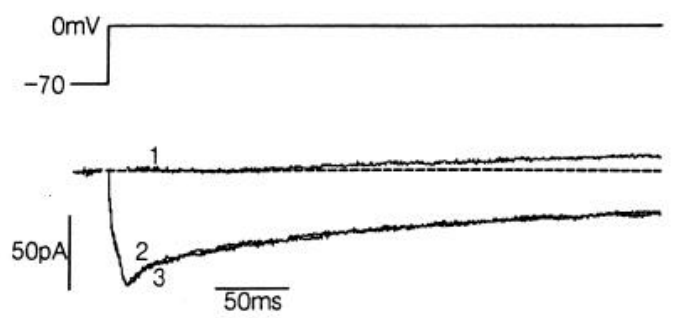

Figure 2. Sharpness of the boundary zone between test and bath solution. $A$, Plot of calcium activity as measured by a calcium-selective microelectrode just above the bottom of a culture dish versus electrode tip position in relation to the test solution stream as shown in Figure 1 . The calcium concentration was $10^{-5} \mathrm{M}$ in the test solution and $10^{-2}$ $\mathrm{M}$ in the bath solution. Dotted lines mark visible borders between solutions. $B$, Voltage-dependent calcium currents activated in a round PC12 cell without processes, by voltage steps from -70 to $0 \mathrm{mV}$. 3 , current recorded under control conditions; 2, current recorded during exposure of the vicinity of the cell to low calcium; 1 , current recorded during exposure of the cell to low calcium. In 1 and 2 , the distance between the visible border zone of the 2 solutions and the cell was 15 $\mu \mathrm{m}$.

ameters of the cell somata and growth cones were done after the experiments under a Zeiss inverted microscope at $375 \times$ magnification (phase-contrast objective $25 \times$, n.a. $=0.45$, ocular $15 \times$ ) using a calibrated eyepiece. In some cases, these measurements were later confirmed on photographs taken after the experiments (see Fig. 6A). To reveal the fine surface structure of the growth cones, high-resolution pictures of sprouting PC12 cells were taken using a Zeiss Axiophot microscope with an oil-immersion phase-contrast objective $(100 \times$, n.a. $=1.3$ ). Currents were recorded in the whole-cell mode of the patchclamp technique (Hamill et al., 1981). Electrodes were pulled from thickwalled Duran glass (Schott Ruhrglas, Mainz, FRG) to give a tip size of ca. $1.5 \mu \mathrm{m}$ and a resistance of around $5 \mathrm{M} \Omega$ when filled with the pipette solution. The electrode's serial resistance was not compensated, because the input resistance of PC12 cells was high (1-10 G $\Omega$ ) and the maximal activated current flow was relatively low $(<500 \mathrm{pA})$. The currents were recorded using a patch-clamp amplifier (see Lux and Brown, 1984), stored on a FM tape recorder at $2500 \mathrm{~Hz}$ and for analysis later digitized using a 12 bit analog-to-digital converter in combination with a LSI 11/ 23 minicomputer. Capacitive currents were reduced by capacitance compensation circuits during experiments. Residual capacitive and leakage currents were later compensated for by adding the mean of 10 20 appropriately scaled responses to hyperpolarizing voltage pulses of 10-50 mV. The cells were normally clamped at holding potentials of -70 or $-80 \mathrm{mV}$ during experiments. For the purpose of steady-state inactivation measurements, the holding potential was temporally changed to different values in some experiments. Currents were activated by depolarizing voltage steps of various sizes, which lasted for $100-400$ msec. Long pulses were applied to record current inactivation. The pulse frequency was low $(\leq 0.05 \mathrm{~Hz})$ to ensure complete recovery from inactivation. Stable recordings could usually be obtained for 10-20 min. 
Laminar flow system. It was crucial in the experiments to apply the test solutions focally to a well-defined area of the cell, without contamination of the bath solution. For this reason, a laminar flow system was developed, based on a multibarrelled pipette described previously (Boll and Lux, 1985; Carbone and Lux, 1987). Five metal needles were aligned and held together with a plastic tube so that the tips of the needles remained uncovered. The tips fitted into a small glass filter funnel whose fine tip (diameter, $50-100 \mu \mathrm{m}$ ) was bent at $45^{\circ}$ to the needles. All 5 needles were used as inflow channels with the flow rate being regulated hydrostatically. To prevent the inflowing solution from dispersing rapidly in the bath solution, a second single-barrelled pipette with a larger tip diameter $(150-200 \mu \mathrm{m})$ was positioned directly opposite the inflow pipette. The outflow rate was driven hydrostatically and could be adjusted by a small clamp. The 2 pipettes were held in such a way that the relative position and the distance between the tips (usually 200-300 $\mu \mathrm{m})$ could be adjusted under the microscope. Once this was done, the laminar flow system could be moved in the bath without any change in the relative position of the tips towards each other. It produced a well-defined stream of test solution with a sharp boundary with the bath solution (see Fig. 1), which could be regulated by adjusting the outflow rate. The test solution was made visible by addition of Fast green ( 2 $\mathrm{mg} / \mathrm{ml}$ ), a stain which did not alter the calcium currents.

Two sets of control experiments were performed to analyze the boundary zone between the test solution and the bath solution. Similar solutions were taken as in the normal experiments, that is, low calcium (ca. $10^{-5} \mathrm{M}$ ) in the test solution (no calcium and no EGTA added), and high calcium $\left(10^{-2} \mathrm{M}\right)$ in the bath solution. The area over which calcium decayed was investigated with a calcium-selective microelectrode, fabricated and calibrated using standard methods (Tsien and Rink, 1980). The electrode was positioned above the bottom of the culture dish and moved in defined steps of $10 \mu \mathrm{m}$ through the test solution stream. As shown in Figure $2 A$, the decay of calcium activity corresponded well with the visible boundaries and was restricted to a thin layer of $20 \mu \mathrm{m}$. This finding was confirmed, when the test solution was positioned 15 $\mu \mathrm{m}$ away from a round PC12 cell, bearing no processes. No change in whole-cell calcium current was recorded, when the test solution was changed from high to low calcium (see Fig. 2B). However, the current completely disappeared, when the cell was $15 \mu \mathrm{m}$ within the test solution. These findings show that it is possible to quantitatively analyze current records from 2 areas of a cell, superfused with 2 different solutions, as long as there is a minimal distance of $30 \mu \mathrm{m}$ between them. An inward current in the low-calcium solution could not be resolved, indicating that the remaining calcium concentration was high enough to prevent the calcium channels from conducting sodium (Carbone and Lux, 1987).

Error estimations. Because growth cone currents were measured in the soma, an error was produced by the electrotonic voltage decay along the neurite between soma and growth cone. This error was estimated using the simple model of a spherical cell with one cylindrical process. The growth cone was simplified as the distal termination of the process. The potential in the soma was considered to be perfectly voltage-clamped at $V_{\mathrm{o}}$. The steady-state departure from a perfect space clamp along the cylinder was calculated using cable equations (Hodgkin and Rushton, 1946; Rall, 1977). Voltage decay along a cylindrical cable follows the equation

$$
\frac{V}{V_{0}}=\frac{\cosh (L-X)+B_{1} \sinh (L-X)}{\cosh (L)+B_{1} \sinh (L)}
$$

with $L=1 / \lambda$ and $X=x / \lambda$ and $l$ being the length of the cylinder. $B_{1}$ is a constant expressing the relation of the conductance terminating the cylinder $G_{1}$ and the virtual conductance of an endless cable at $X=L$ $\left(G_{\alpha}\right)$. With

$$
G_{\infty}=\pi d /\left(2 R_{m}\right) \sqrt{\left(R_{m} / R_{i}\right) d}
$$

and

$$
G_{1}=\pi d^{2} / R_{m}
$$

$R_{m}$ being the resistance of the membrane, $R_{i}$ the resistivity of the core (cytoplasma), and $d$ the diameter of the neurite, and assuming a 4 times lower membrane resistance in the growth cone than in the neuritic shaft, $B$, becomes

$$
B_{1}=2 \sqrt{\left(R_{i} / R_{m}\right) d}
$$

With $R_{m}=3500 \Omega \mathrm{cm}^{2}$ (mean value taken from our experiments during the peak calcium current flow), $R_{i}=70 \Omega \mathrm{cm}$ (Rall, 1977 , cat motoneurons) and $d=2 \mu \mathrm{m}$, voltage decays to $95 \%$ of the initial value within an electrotonic length of 0.3 , corresponding to a neuritic length of 150 $\mu \mathrm{m}$. Thus, during a voltage step from -80 to $10 \mathrm{mV}$ as was applied in the experiment illustrated in Figure 3 to obtain maximum current flow, an error of $4.5 \mathrm{mV}$ is expected in a growth cone $150 \mu \mathrm{m}$ apart from the soma. Such an error would produce a diminution of the growth cone current by $5-15 \%$ (Fig. 3C).

A second error is produced by the current loss between the site of conductance change (growth cone) and current recording (soma). This error can be estimated by a comparison of the conductance change at the end of a cylinder $\left(\Delta G_{1}\right)$ to the change produced in total input conductance of the cylinder $\left(\Delta G_{0}\right)$. The total input conductance of a cylinder is expressed as follows:

$$
G_{0}=\frac{B_{1}+\tanh (L)}{1+B_{1} \tanh (L)} G_{\infty}
$$

A conductance change at the end of a cylinder can be expressed by a change in the boundary constant $B_{1}$. The increase in input conductance due to a 10 times increase in conductance at the end of the cylinder is then

$$
\Delta G_{0}=\pi d /\left(2 R_{m}\right) \sqrt{R_{m} / R_{i} \cdot d}\left(\frac{10 B_{1}+\tanh (L)}{1+10 B_{1} \tanh (L)}-\frac{B_{1}+\tanh (L)}{1+B_{1} \tanh (L)}\right)
$$

with $\Delta G_{1}=9 \pi d^{2} / R_{m}(3)$ and $L=0.3: \Delta G_{0} / \Delta G_{1}=0.90$. Thus, in short neurites $(<150 \mu \mathrm{m})$, more than $90 \%$ of the current produced by a conductance change in the growth cone is recorded in the soma.

\section{Results}

In PC1 2 cells voltage-dependent calcium currents appeared with pulses to -20 or $-10 \mathrm{mV}$ and reached maximal values between 0 and $+20 \mathrm{mV}$ (Streit and Lux, 1987). They resembled the classical high-voltage activated currents described in several neuronal cells (Carbone and Lux, 1984; Nowycky et al., 1985; Yaari et al., 1987). The peak amplitudes of the currents varied between 10 and $500 \mathrm{pA}$ in $10 \mathrm{~mm}$ calcium. Both current-voltage relationships and peak amplitudes depended on whether and for how long the cells were treated with NGF. Cells exposed to NGF for several days had larger currents which appeared at more negative potentials than did cells not treated with NGF. Voltage-dependent calcium currents were also recorded from growth cones of NGF-treated PC12 cells. A quantitative comparison between calcium currents from the soma and the growth cone of individual intact cells was, however, not possible because the currents recorded when the patch pipette was positioned on growth cones were considerably contaminated by currents originating in the soma. In Figure 3 an experiment is illustrated where calcium currents were recorded first from the growth cone and then from the soma of the same $\mathrm{PCl} 2$ cell. The amplitudes of the currents, as well as the current-voltage relationships were quite similar at both locations of recording. However, while the currents recorded on the soma seemed to be reasonably well clamped (smooth time course, no deviation from voltage clamp at voltages where calcium currents became activated), the currents recorded from the growth cone clearly showed signs of imperfect voltage clamp. This experiment thus illustrates that currents from central parts of the cell, where the voltage clamp is insufficient, contribute to the total current recorded in the growth cone. Growth cone currents will also be expected to contribute to the whole-cell current recorded in the soma. Because these currents are small compared with the wholecell currents due to the small size of the growth cones, and because the voltage attenuation from a growth cone to the soma is greater than that from the soma to the growth cone due to 
A
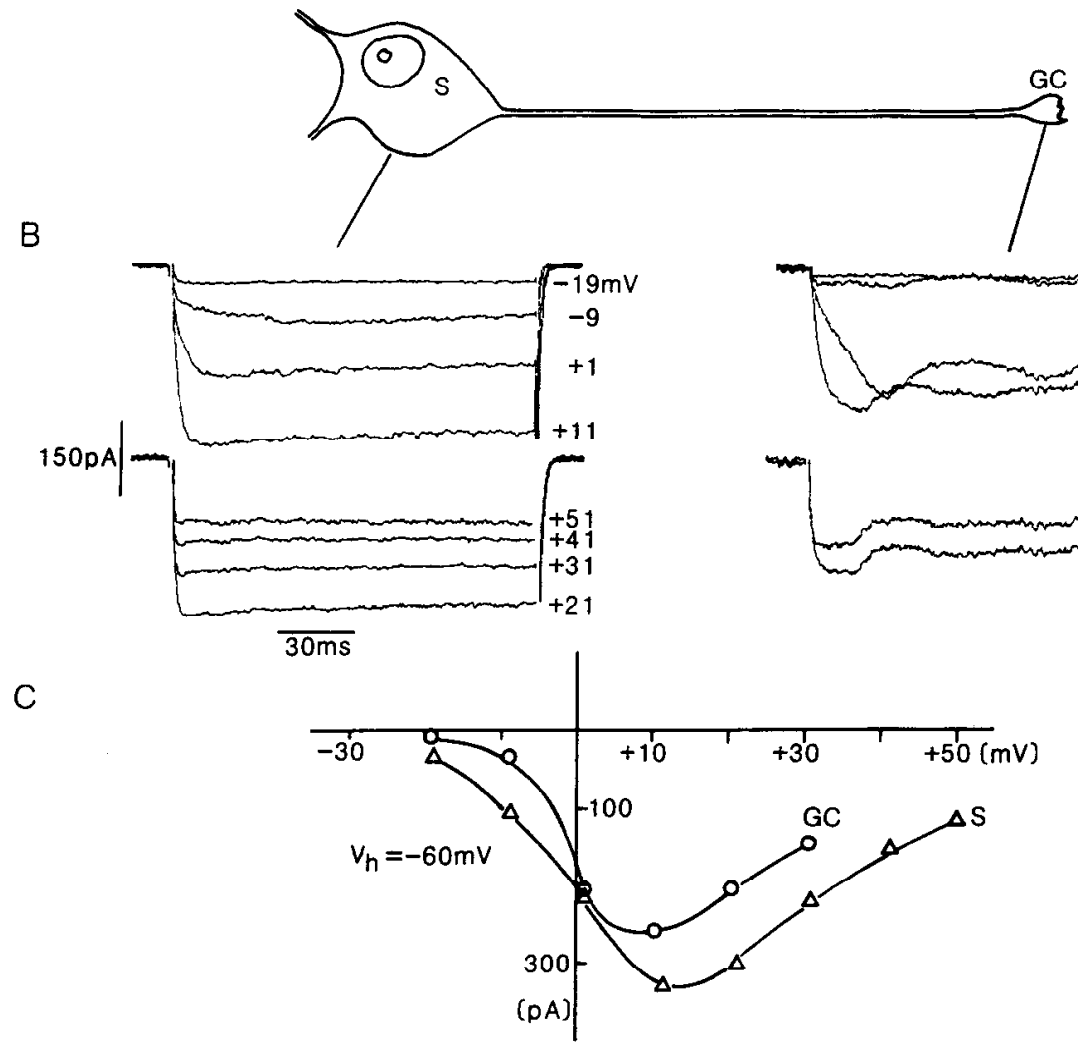

Figure 3. Voltage-dependent calcium currents recorded first from the growth cone and then from the soma of a $\mathrm{PC} 12$ cell. $A$, Schematic drawing of the $\mathrm{PC} 12$ cell, grown in the presence of NGF for $7 \mathrm{~d}$. B, Calcium currents activated in the growth cone (right) and in the soma (left) by voltage steps from a holding potential of $-60 \mathrm{mV}$ to potentials between -19 and $51 \mathrm{mV}$. The 2 sets of currents were recorded within a time domain of $20 \mathrm{~min}$. C, Current-voltage relationships of the currents shown un$\operatorname{der} B$. the relatively low impedance of the soma (Johnston and Brown, 1983), the contribution of growth cone currents to whole-cell currents does not produce the same irregularities in time course that are seen in the growth cone recordings. A quantitative comparison between soma and growth cone portions of currents in individual cells seemed therefore to be possible, if the growth cone currents were large enough to be separated properly from the soma current and if the voltage and current errors induced by the electrotonic distance between growth cone and soma were small enough to be neglected. The second condition is fulfilled in cells with neurites of short electrotonic length. Applying cable equations (Hodgkin and Rushton, 1946; Rall, 1977) to short neurites, growth cones were estimated to be isopotential with the soma with an error of less than $5 \%$, as long as the electrotonic length of the neurite is below 0.3 . Under such conditions the attenuation of current along the neurite was shown to be below $10 \%$ of the initial value. Such electronic lengths are represented by neurites 100-200 $\mu \mathrm{m}$ long with diameters between 2 and 3 $\mu \mathrm{m}$. Such processes were long enough to allow satisfactory separation of growth cone currents from soma currents by focally supprcssing calcium currents with a laminar flow of low-calcium solution. Residual ionic currents in the low-calcium solution were small compared with the calcium currents and routinely subtracted.

Cells with short enough neurites could be obtained during the first 5 days in the presence of NGF. Neurite outgrowth started within the first $48 \mathrm{hr}$ of NGF treatment and proceeded in the presence of NGF at a slow rate of around $30 \mu \mathrm{m} / \mathrm{d}$. Cells with sprouts of $40-80 \mu \mathrm{m}$ were selected to study the calcium current density in freshly sprouting growth cones. To reveal the calcium current contribution of growth cones in such cells, voltage steps producing maximum current flow were applied to the cells at a low frequency, and constant-current amplitudes were obtained (Fig. 4). Then the growth cone of a cell was exposed to a laminar flow of control solution. No change in the recorded whole-cell current was usually recorded under such conditions. When then the laminar flow solution was changed to low calcium, a reduction of the current amplitude by 10-50\% occurred. When the whole cell was exposed to low calcium, the current usually disappeared completely (see Fig. $2 B$ ). Unfortunately, neither the reduction of the currents during exposure of the growth cones nor the disappearance of the currents during exposure of whole cells to low calcium was fully reversible in normal bath solution containing calcium or barium. The reason for this was not further investigated because the lack of reversibility would not negate our results concerning the distribution of calcium currents, as long as it could be shown that the reduction of the current was due to the omission of calcium conductances in the growth cone or distal neurite and not at another site. As pointed out in the method section, the visible border zone between bath solution and test solution corresponded well with the area of decay of calcium activity when the test solution contained low calcium. The experiments were done under visual control to exclude the possibility that other areas were unintentionally exposed to low calcium. This control was not possible using cadmium (1 mM) to block the calcium currents reversibly (experiments not shown). Since cadmium substantially reduces calcium currents at concentrations much lower than $1 \mathrm{~mm}$, an effect of cadmium on other areas could not be cxcluded in these experiments.

To minimize influences of calcium current run down to the differences between subsequent recordings, constant-current 
A
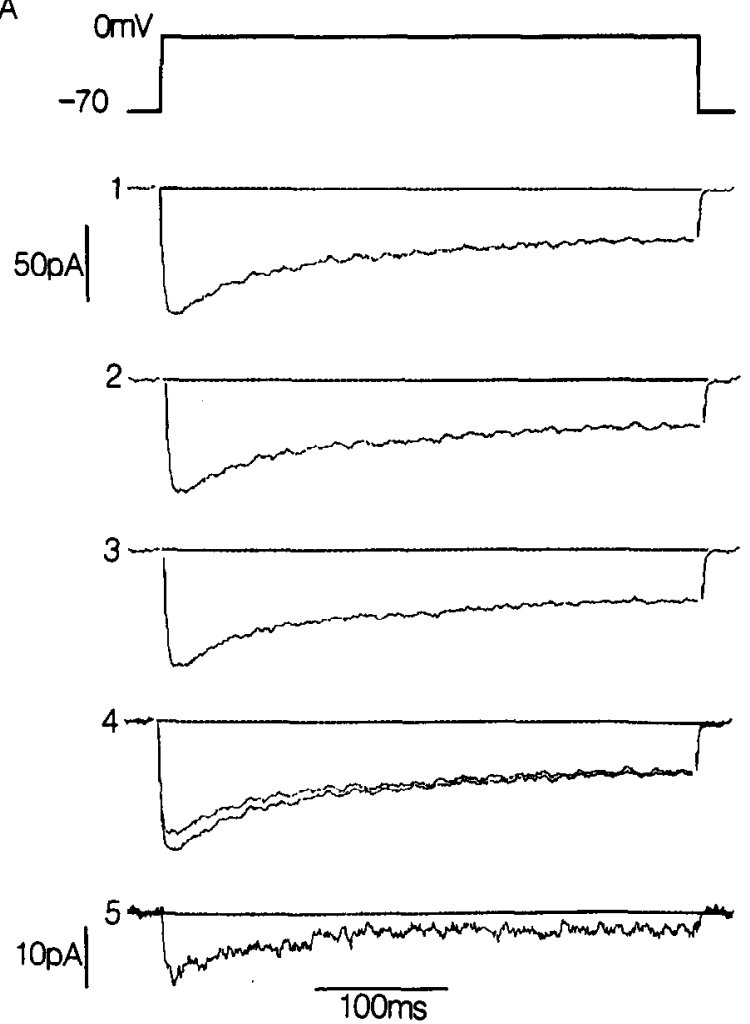

B

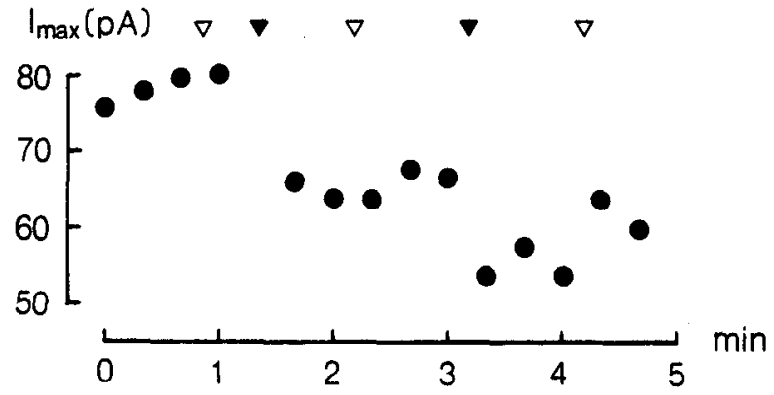

Figure 4. Decrease of whole-cell calcium current during exposure of the growth cone of a sprouting PC12 cell to low-calcium solution. The cell was treated with NGF for $2 \mathrm{~d}$, the growth cone bearing neurite was $50 \mu \mathrm{m}$ long. $A$, Peak calcium currents activated by voltage steps from -70 to $0 \mathrm{mV}: 1$, during exposure of the growth cone to a test solution containing normal calcium (10 mM); 2 , during exposure of the growth cone to low-calcium solution, and 3 , during reexposure to normal calcium solution; 4 , superposition of trace 1 and $2 ; 5$, scaled up difference between 1 and 2 . B. Plot of the peak current amplitudes recorded in this cell versus time. Open triangles indicate the onset of the superfusion of the growth cone with control solution; filled triangles, the onset of low-calcium exposure. Pulses were applied 1/20 sec.

amplitudes during control conditions were first obtained and then the records immediately before and after low-calcium exposure of the growth cone were used to calculate the growth cone current by subtraction. Alternatively, growth cone currents were recorded directly during exposure of a cell to low calcium while leaving the growth cone in normal bath solution (Fig. $5 \mathrm{~A}$, trace 6). Recordings obtained during exposure of the entire cell to low calcium were routinely subtracted from these currents.

Calcium currents of the cells treated with NGF for a maximum of $48 \mathrm{hr}$ which had short processes and were therefore
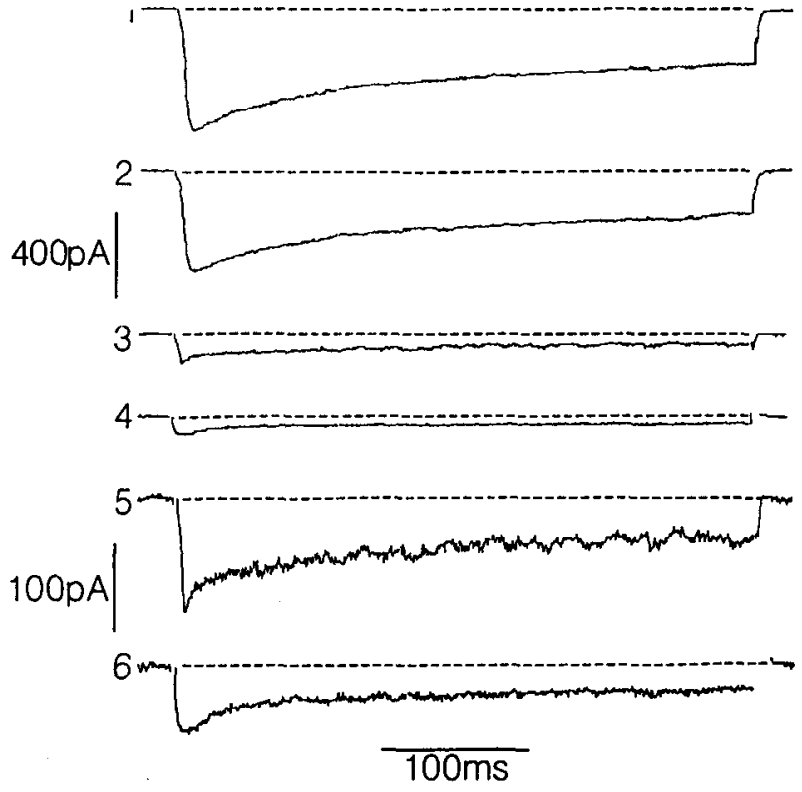

Figure 5. Barium currents originating from the growth cone of a sprouting PC1 2 cell. The primed cell ( $10 \mathrm{~d}$ with NGF) was in the present passage for $2 \mathrm{~d}$. The bath solution contained $50 \mathrm{~mm}$ barium. First, the growth cone and then the rest of the cell without that growth cone were exposed to the solution containing low calcium. 1, Current activated by voltage steps from -80 to $0 \mathrm{mV}$ in normal bath solution; 2 , current recorded during low-calcium exposure of the growth cone, soma current; 3 , difference between 1 and 2 , growth cone current; 4 , current recorded during low-calcium exposure of the whole cell without the growth cone (growth cone current); 5 and 6 , traces 3 and 4 at higher magnification.

selected for these experiments were small (10-50 pA). To increase the current amplitudes and thereby improve the resolution for growth cone contributions, the cells were pretreated with NGF for $8 \mathrm{~d}$ before the passage. After subculturing, processes started to regrow within the first $24 \mathrm{hr}$ in the presence of NGF. Such "primed" cells (Greene and Tischler, 1982) had considerably larger calcium currents during the initial phase of neurite growth. To further increase current amplitudes, barium (50 mM) was used as a charge carrier. Under such conditions peak current amplitudes were in the range of 100-500 pA. Figure 5 shows records from a primed cell of the second day in passage. When the growth cone of this cell was exposed to low calcium, the whole-cell current was reduced by maximum $100 \mathrm{pA}$. When the whole cell except the growth cone was exposed to low calcium, a current with a peak amplitude of 72 pA was recorded, obviously originating in the growth cone. The time courses of the 2 currents (Fig. 5, traces 5 and 6) were similar, after 200 msec $50 \%$ of the initial peak current was inactivated in trace 5 and $48 \%$ in trace 6 . However, the current recorded during exposure of the soma to low calcium (trace 6) was considerably smaller (by $31 \%$ ) than that recorded during exposure of the growth cone (trace 5). This finding can be explained by the fact that the growth cone was exposed to low calcium first, and then the laminar flow system was moved to superfuse the soma. Therefore, trace 6 represents current which had recovered from previous exposure to low calcium. As mentioned before, the effect of low-calcium exposure was not fully reversible. To obtain a measure of the current densities in the cell segments, the mean of the recorded peak currents in the 2 situations was divided by the visible surface area of the segments. For an 
A
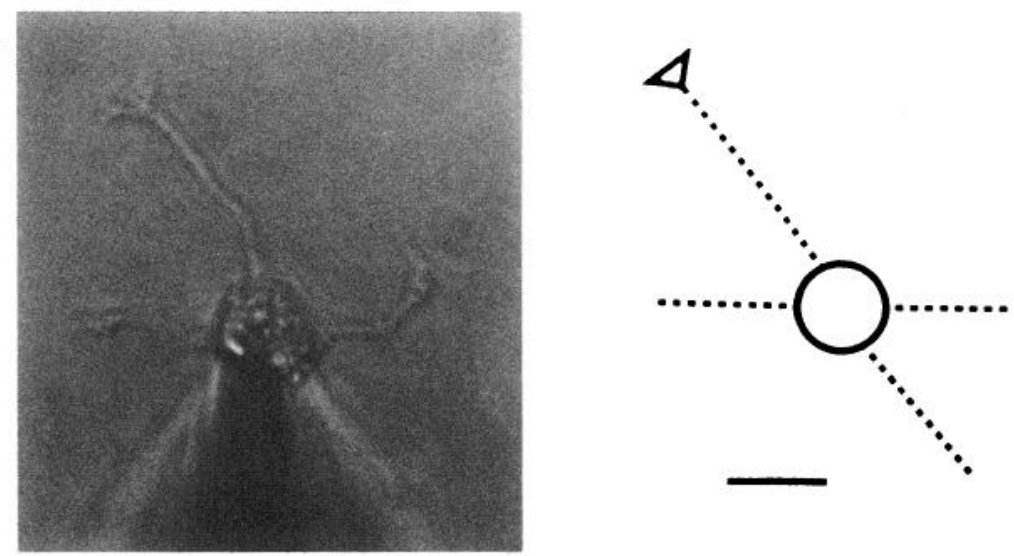

B

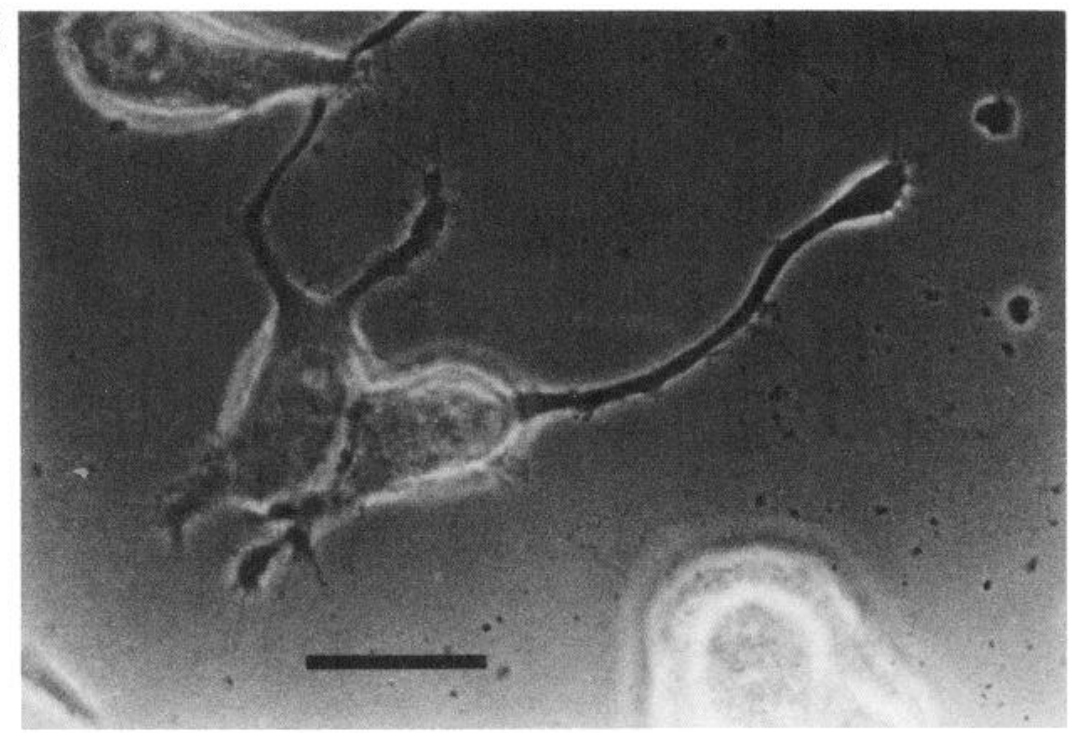

Figure 6. Morphology of sprouting PC12 cells. A, Photograph of the cell from which the currents shown in Figure 5 were recorded, taken after the experiment (phase-contrast microscopy, objective $25 \times$, n.a. $=0.45$ ). The low resolution is partly due to the vibration of the patch pipette and the cell during the shutter movements. The geometrical model fitted to this cell for an estimate of the surface areas of the investigated growth cone (upper left) and the soma is shown at right. The neurites (dotted lines) were neglected when the current densities were calculated. Scale bar, $20 \mu \mathrm{m}$. $B$, High-resolution photograph of a living PC12 cell grown for 2 $d$ in the presence of NGF (phase-contrast microscopy, oil-immersion objective $100 \times$, n.a. $=1.3$ ). To take the photograph the cell was kept in normal bath solution containing no NGF, to obtain identical conditions as used in the experiments. Note the fine filopodia and lamellipodia at the leading edge of the growth cone, along the neuritic shaft and also at some locations of the soma. Scale bar, $20 \mu \mathrm{m}$. estimate of the surface areas of the segments, the diameters of somata and growth cones were measured directly following the experiments using a calibrated eyepiece. In Figure $6 \mathrm{~A}$ a photograph of the cell, taken after the experiment illustrated in Figure 5, is shown together with a schematic drawing of the same cell. The membrane surface of the growth cone and the soma were estimated by fitting them to simple geometrical models. In this example, the growth cone was fitted by an equilateral triangle with a base of $13 \mu \mathrm{m}$ and an amplitude of $7 \mu \mathrm{m}$. The soma was fitted to a hemisphere with a diameter of $20 \mu \mathrm{m}$. The surfaces of these models $\left(46 \mu \mathrm{m}^{2}\right.$ for the growth cone and $628 \mu \mathrm{m}^{2}$ for the soma) were used for the calculation of the current densities. Neuritic shafts were fitted as cylinders with a mean diameter of $2 \mu \mathrm{m}$. In this and all other experiments, where the interest was focused on the growth cones, neither the neuritic shafts nor the growth cones of additional neurites were taken into account when calculating the current density, for the current densities of neuritic shafts were shown to be small in separate experiments (see Fig. 8). This procedure to estimate the surface area of the cells obviously neglected the fine membraneous structures of the surface like lammelipodia and filopodia. As shown in Figure $6 B$, these structures can be visualized by use of highresolution oil-immersion microscopy. Lammelipodia and filopodia were most frequently seen at the leading edge of growth cones, but they were also found at neuritic shafts and somata. However, they only minimally increased the membrane surface of a growth cone compared with the membrane surface of the soma. The error introduced by neglecting these membrane rufflings was estimated in the example shown by measuring the surface area of the visible lamellipodia and filopodia assuming a diameter of the small filopodia of $0.2 \mu \mathrm{m}$. The total areas of these structures on the growth cone and the soma were then added to the surfaces of the growth cone and the soma obtained by fitting them to geometrical models (in this case an ellipse for the growth cone and an elliptic hemisphere for the soma). The surface of the growth cone normalized to the surface of the soma was 0.10 when the filopodia and lamellipodia were neglected and 0.13 when they were taken into account. Thus, the use of the simplified models to calculate the current densities in this example would overestimate the normalized current density of the growth cone by $30 \%$.

Based on the simplified models the current density of the 


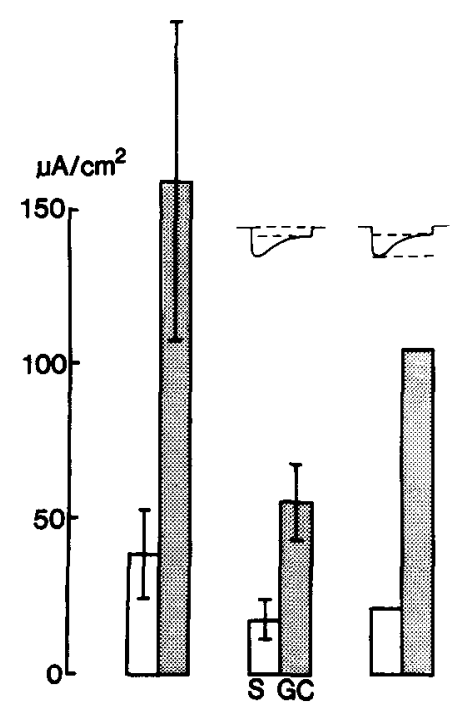

Figure 7. Densities of peak currents, inactivating current components and persistent currents in somata and growth cones of sprouting PC12 cells. Barium, $50 \mathrm{~mm}$, was used as charge carrier in all experiments. Open bars represent mean values of somata, dotted bars, mean values of growth cones ( \pm SEM, $n=6$ ). Inactivating and persistent portions of current were separated after $200 \mathrm{msec}$ pulse duration.

growth cone illustrated in Figures 5 and $6 A$ was $187 \mu \mathrm{A} / \mathrm{cm}^{2}$ compared with a current density of the soma of $73 \mu \mathrm{A} / \mathrm{cm}^{2}$. Current densities proved to be higher than in the somata in all growth cones investigated $(n=11)$. The mean current density of the growth cones normalized to that of the somata in individual cells was $5.4 \pm 1.2( \pm \mathrm{SEM}, n=7)$. Furthermore, comparing the mean peak current densities of 6 experiments performed under identical conditions (primed cells with $50 \mathrm{mM} \mathrm{Ba}$ in the external solution) to the mean current densities after 200 msec pulse duration (Fig. 7), it was found that current inactivation was more pronounced in growth cones than in somata.

An additional point of interest was the current density along the shafts of the neurites. For this purpose, cells with slightly longer neurites (80-200 $\mu \mathrm{m})$ were selectively investigated. Figure 8 shows an experiment where the growth cone was first exposed to low calcium to record its current contribution. Then the laminar flow system was moved towards the soma in 3 steps of $15 \mu \mathrm{m}$. At each position the whole-cell calcium current was recorded. In this way the current contributions of 3 segments of the neuritic shaft could be recorded by subtraction of the whole cell currents. A considerable current was revealed in the growth cone, a small one in the proximal segment, and no current in the 2 intermediate segments of the neurite, strongly suggesting that in sprouting $\mathrm{PCl} 2$ neurites the calcium channel density along the neuritic shaft is very low. This finding was confirmed in other experiments where 100 - to $200-\mu \mathrm{m}$-long segments of neurites with more distant growth cones were exposed to low calcium without measuring a decrease in wholecell currents.

Small currents, however, were routinely detected on very proximal segments of neurites (see Fig. 8, trace 9). The length of these segments depended on whether primed cells were used for the experiment. Primed cells grew neurites 80-200 $\mu \mathrm{m}$ long, which were selected for these experiments, in 1-3 d (Fig. 8 shows an example of a primed cell). Cells with neurites of similar length were found only after $4-8 \mathrm{~d}$ when immature cells were treated

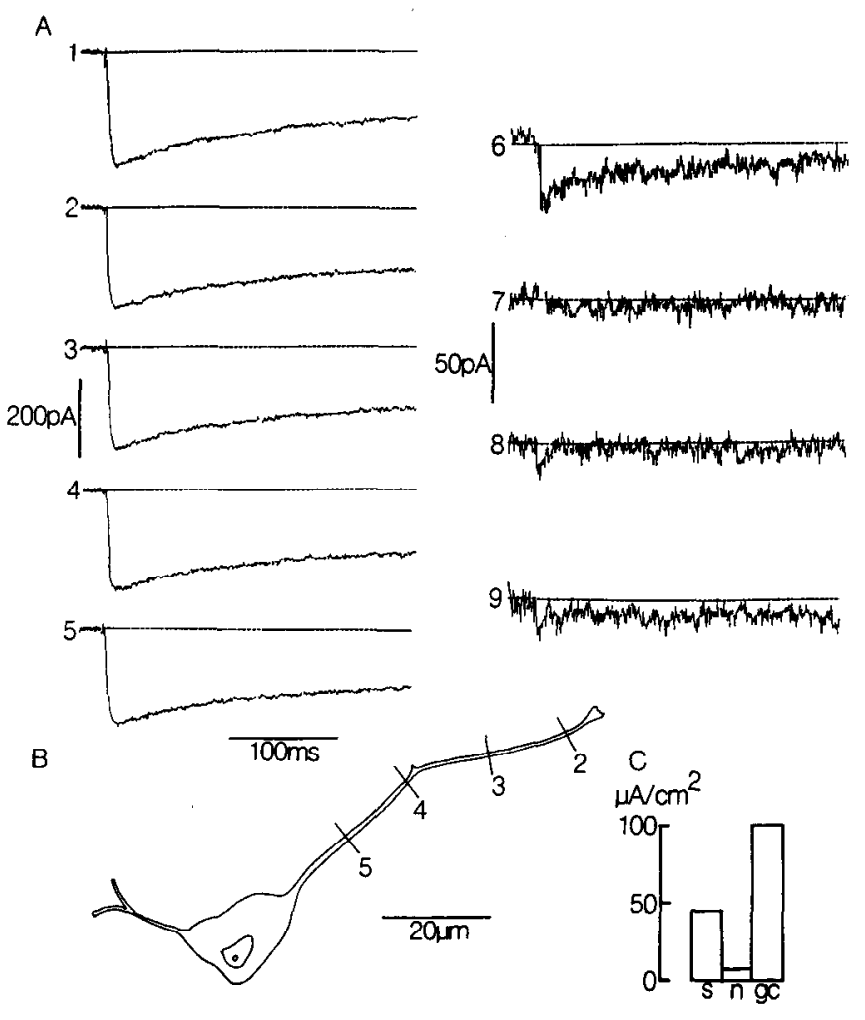

Figure 8. Calcium channel density on the neuritic shaft of a sprouting PC1 2 cell. Primed cell ( $10 \mathrm{~d}$ with NGF), second day in the present passage. $A$, Whole-cell barium currents activated by voltage steps from -80 to $0 \mathrm{mV}: 1$, control condition; 2 , during low-calcium exposure of the growth cone; $3-5$, during low-calcium exposure of distal segments of the neurite of increasing lengths. The positions of the border zone between test and bath solution corresponding to the current records is shown under $B$. 6 , Difference between 1 and 2 , growth cone current; 7 , difference between 2 and 3 , current from the distal segment of the neurite; 8 , difference between 3 and 4 , current from the intermediate segment; 9 , current from the proximal segment. $B$, Drawing of the microscopic display of the cell. $C$, Bars representing current densities from the soma, neuritic shaft, and growth cone of this cell. The mean of the 3 records was taken to calculate the current density of the neurite.

with NGF for the first time, since neurite growth was much slower in such cells. In such slowly growing cells, calcium currents were detected in longer proximal segments of neurites. Figure 9 shows an example of such a cell where the same calcium current density as in the soma was detected on a segment 40 $\mu \mathrm{m}$ away from the soma. A much smaller current density was revealed in the more distal segment of the neuritic shaft, indicating a somatofugal decrease in current density in this segment, which is more then proportional to the decrease expected from cable equations. Occasionally, short neurites whose growth had stopped were found in older cultures where the current density of the entire neuritic shaft was in the range of that of the soma. These findings suggest a growth rate dependence of the somatofugal decay in calcium current density along neurites.

\section{Discussion}

\section{Current-density measurements}

In the present work, calcium current contributions of growth cones and neurites of $\mathrm{PC} 12$ cells were measured by recording changes in whole-cell current in the soma, caused by eliminating current components generated in the neurites or growth cones. It was shown in control experiments and by comparing different 
experimental protocols, that such changes in whole-cell current could not be attributed to nonspecific effects such as contamination of the bath solution with low-calcium solution or run down of calcium currents. Growth cones and neurites cannot be regarded as isopotential with the soma in voltage-clamp experiments due to their electrotonic distance from the soma. In addition, currents originating in the periphery do not fully appear in the soma. Such errors were kept minimal by limiting the analysis to short processes and by applying voltage pulses producing maximal current flow. With this protocol, current originating in the periphery of the cells are underestimated in our analysis by $10-30 \%$. Furthermore, in all cells the current remaining after subtraction of the growth cone current was attributed to the soma, neglecting the contributions of growth cones of additional neurites and neuritic shafts. Because all these errors underestimate the amplitude of the growth cone current compared with the soma current, the high current density in the growth cone cannot be attributed to them.

To compare the current densities in the center and the periphery of the cell, a measure of membrane surface contributions of the somata, neurites, and growth cones had to be obtained. Morphometric measurements were used to calculate the membrane area based on simplified geometrical models, such as hemispheres for somata, triangles, ellipses, or rectangles for growth cones and cylinders for neurites. Surface ruffles, described in processes and somata of PC12 cells (Jacobs and Stevens, 1986), were neglected. Current densities of growth cones might therefore be overestimated as a consequence of a more pronounced membrane ruffling in growth cones (Connolly et al., 1987). As pointed out in the Results section, based on the analysis of a high-resolution photograph of a sprouting PC12 cell, the overestimation introduced in the ratio of growth cone to soma current density by neglecting filopodia and lamellipodia is in the range of $30 \%$. This estimate is supported by results from mechanically isolated growth cones (Streit and Lux, 1987). In these experiments, the membrane surface was estimated by the morphometric method, as well as by measuring the membrane capacitance of isolated growth cones and cell bodies. When the mean membrane area of the growth cones was normalized by the mean membrane area of the cell bodies, the value obtained by the capacitance method was $28 \%$ higher than that obtained by the morphometric method.

In 3-dimensional serial electron microscopic reconstructions of freshly sprouting cells, somata and neurites as well as growth cones show a highly irregular shape (Jacobs and Stevens, 1986). Furthermore, according to Connolly et al. (1987), not all of the observed growth cones possessed ruffles, and those which had, lost them when NGF was withdrawn for several hours. Our experiments were done in growth cones which did not show further sprouting, after withdrawal of NGF for 10-90 min, a protocol which might have reduced the ruffling of the growth cone membrane. We conclude, therefore, that the observed differences in current density cannot be fully attributed to an underestimation of the membrane surface of the growth cones compared with neuritic shafts and somata.

\section{Calcium channel distribution during neurite growth}

The results indicate that calcium channels are distributed in growing PC12 cells in a highly specific way. The highest channel density is found in the growth cones, whereas only few channels are present along the developing neurite. Growth cones represent the location where new membrane is incorporated in the
A a

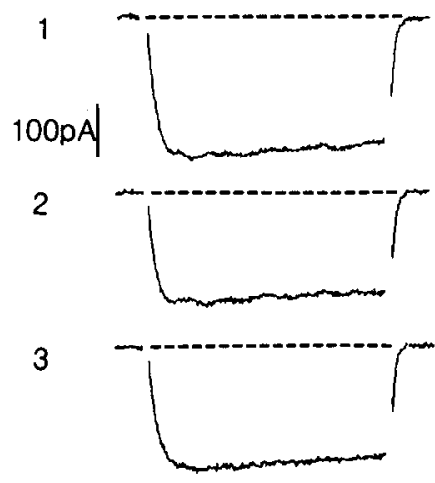

4

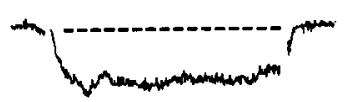

$50 \mathrm{pA}$

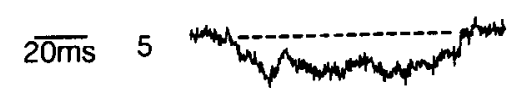

B

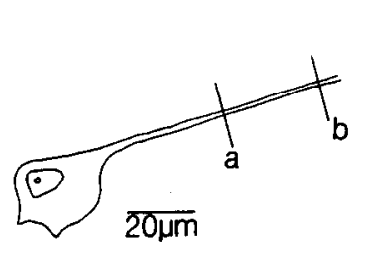

b
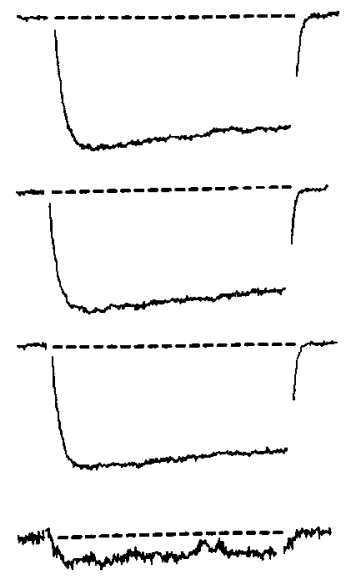

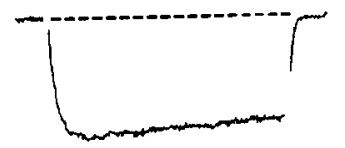

C $\quad \mu \mathrm{A} / \mathrm{cm}^{2}$

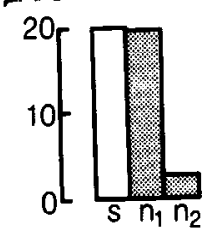

Figure 9. Calcium channel density on the proximal segment of a neurite. Cell treated with NGF for $8 \mathrm{~d}, A$, Calcium currents activated by voltage steps from -70 to $0 \mathrm{mV}: 1$, control; 2, exposure of the distal neurite to low calcium; 3 , reexposure to control solution; 4 , difference between 1 and 2 , current originating from the distal part of the neurite. The experiment was performed with 2 positions of the laminar flow system $(a$ and $b$ ), the 2 border zones between solutions are indicated under $B$. 5, Difference between $4 a$ and $4 b$, current originating from the neurite segment between $a$ and $b$. $C$, Bars representing current densities of the soma including the proximal segment of the neurite(s), the neurite segment between $a$ and $b\left(n_{1}\right)$ and the segment distal of $b\left(n_{2}, 150 \mu \mathrm{m}\right.$ long).

growing neurite (for review, see Landis, 1983). New membrane is probably transported to the growth cones in the form of vesicles. The high calcium current density in the growth cones suggests that calcium channels are already incorporated in such vesicles. If this is true, the calcium channel density of the most distal segments of the neuritic shaft neighboring the growth cone should be in the range of that of the growth cone, with a decay of the channel density in a distal to proximal direction depending on the turnover rate of channels in the membrane. However, the current density in the most distal segment of the neuritic shaft was found to be very low, as in the rest of the shaft (see Fig. 8). A gradient in calcium activity following membrane depolarization, between growth cone and distal neuritic shaft was also found in other neuronal cells using calcium-sensitive dyes (Bolsover and Spector, 1986; Connor, 1986). The hypothesis therefore implies that the turnover rate of the channels in the growth cone is very fast. In a second hypothesis, clusters of calcium channels with a slow turnover rate are maintained in the growth cones from the beginning of neurite growth on. This hypothesis implies that calcium channels are fixed in the growth cone since otherwise they would not migrate with the growth 
cone (see Bray, 1970), and that the new membrane incorporated in the growth cone does not contain calcium channels. Binding of clustered sodium channels to the cytoskeleton has been recently reported (Srinivasan et al., 1988). The idea of incorporation of membrane without calcium channels during neurite growth is favored by previous results, which are described in detail in our previous paper (Streit and Lux, 1987). Initial NGFinduced soma growth and sprouting of PC12 cells is not accompanied by an increase in whole-cell calcium current, thus leading to a decrease in calcium current density in such cells. With prolonged NGF treatment, the calcium currents increase in amplitude while soma growth and outgrowth of neurites had ceased. This finding was interpreted as a temporal delay between membrane growth and channel incorporation. Furthermore, calcium current density in growth cones was constantly high from the beginning of neurite outgrowth without any further increase with prolonged NGF treatment. All these observations support the hypothesis that calcium channel clusters, preexisting at sprouting sides of $\mathrm{PC} 12$ cells, are fixed in the growth cones throughout neurite elongation, whereas new membrane is added without calcium channels. The new membrane of the soma and neuritic shafts is later equipped with newly synthesized calcium channels from the soma, as indicated by the increasing whole-cell currents during prolonged NGF treatment and on proximal neuritic shafts by the increasing calcium current density towards the soma. Comparing neurites of similar length but of different ages (different growth rates), the proximal segments of neurites, where a considerable calcium channel density was detected, were longer in slow-growing (elder) than in fast-growing neurites. This indicates that the calcium channels of the neuritic shafts come from the soma after the neurite has formed. With prolonged NGF treatment ( $>10 \mathrm{~d}$ ) and consolidation of at least the proximal parts of the neurites, the calcium current density of somata and neurites approached that of growth cones. This hypothesis is supported by findings of Small and Pfenninger (1984) in olfactory axons of the bullfrog. They described a decreasing gradient of intramembrane particles along the somatofugal axis of a growing axon versus a uniform distribution of these particles in the mature axon. However, in growth cones they found discrete domains of high and low particle densities, giving together a higher mean density than the neighboring axonal shaft. Rather high densities of calcium conductances are also described for dendritic trees of various parts of the mature CNS (Llinás and Sugimori, 1980; Llinás and Yarom, 1981) and for hippocampal cells maintained in culture for 4-6 weeks (Bley et al., 1987; Yaari et al., 1987).

\section{Increased inactivation of growth cone currents}

Calcium current contributions from growth cones inactivated more during $400 \mathrm{msec}$ pulses than soma currents. This observation cannot be explained by a deformation due to the recording of growth cone currents in the soma, since this would produce an increase rather than a decrease of the time constant of current inactivation. In line with this view, current contributions of the soma showed less inactivation than the growth cone current when recording from the growth cone (Streit and Lux, unpublished observations). Marked inactivation therefore seems to be an attribute of calcium channels in the growth cone, possibly as a consequence of channel clustering. It might also be interpreted in terms of a specific distribution of 2 classes of calcium channels (Miller, 1987; Kongsamut and Miller, 1986; Friel and Tsien, 1988; Plummer et al., 1989). A more detailed interpretation of this finding will be given in a forthcoming paper.

\section{Possible role of calcium channel distribution}

The concentration of calcium channels in the growth cones of PC12 cells implies a function of these channcls, which is most probably related to the regulation of neurite growth. In fact, the importance of calcium activity in the growth cone for its motility and shape, attachment to the substrate and neurite elongation has been demonstrated many times (Schubert et al., 1978; Fukuda and Kameyama, 1979; Willard, 1980; Meiri et al., 1981; Anglister et al., 1982; Mattson and Kater, 1987). However, if voltage-dependent calcium channels are indeed involved in neurite growth regulation in PC12 cells, it is an open question how these currents are activated during growth. The membrane potential of growth cones seems to be quite stable in a range where calcium currents are not activated, -40 to $-50 \mathrm{mV}$ (O'Lague et al., 1985; Streit and Lux, unpublished observations). A possible explanation has been given by Freeman et al. (1985): small amounts of leakage currents through voltage-dependent calcium channels might produce an endogenous electrical field due to the uneven distribution of the channels, which might play a role in the fusion of new membrane or in the transport of cellular particles responsible for growth. They describe such an endogenous electrical field in growth cones of goldfish retinal ganglion cells, which seemed to be generated by calcium currents. In line with this hypothesis, high concentrations of intracellular free calcium were found in the tips of growing neuronal sprouts (Connor, 1986). In addition, externally applied electrical fields are reported to be able to guide the growth of neurites towards the cathode (for review, see Landis, 1983).

To summarize, our results show that calcium channels are actively concentrated at the tip of outgrowing neurites of $\mathrm{PC1} 2$ cells, suggesting a role of these channels in the regulation of neurite elongation.

\section{References}

Anglister, L., I. C. Farber, A. Shahar, and A. Grinvald (1982) Localization of voltage-sensitive calcium channels along developing neurites: Their possible role in regulating neurite elongation. Dev. Biol. 94: 351-365.

Belardetti, F., S. Schacher, and S. A. Siegelbaum (1986) Action potentials, macroscopic and single channel currents recorded from growth cones of Aplysia neurons in culture. J. Physiol. (Lond.) 374: 289-313.

Bley, K. R., D. V. Madison, and R. W. Tsien (1987) Multiple types of calcium channels in hippocampal neurons: Characterization and localization. Soc. Neurosci. Abstr. 13: 1010.

Boll, W., and H. D. Lux (1985) Action of organic antagonists on neuronal calcium currents. Neurosci. Lett. 56: 335-339.

Bolsover, S. R., and I. Spector (1986) Measurements of calcium transients in the soma, neurite, and growth cone of single cultured neurons. J. Neurosci. 6: 1934-1940.

Bray, D. (1970) Surface movements during the growth of single explanted neurons. Proc. Natl. Acad. Sci. USA 65: 905-910.

Carbone, E., and H. D. Lux (1984) A low voltage-activated, fully inactivating $\mathrm{Ca}$ channel in vertebrate sensory neurones. Nature 310 : 501-502.

Carbone, E., and H. D. Lux (1987) Kinetics and selectivity of a lowvoltage-activated calcium current in chick and rat sensory neurones. J. Physiol. (Lond.) 386: 547-570.

Cohan, C. S., J. A. Connor, and S. B. Kater (1987) Electrically and chemically mediated increases in intracellular calcium in neuronal growth concs. J. Ncurosci. 7: 3588-3599.

Connolly, J. L., P. J. Seeley, and L. A. Greene (1987) Rapid regulation of neuronal growth cone shape and surface morphology by nerve growth factor. Neurochem. Res. 12: 861-868.

Connor, J. A. (1986) Digital imaging of free calcium changes and of 
spatial gradients in growing processes in single, mammalian central nervous system cells. Proc. Natl. Acad. Sci. USA 83: 6179-6183.

Freeman, J. A., P. B. Manis, G. J. Snipes, B. N. Mayes, P. C. Samson, J. P. Wikswo, and D. B. Freeman (1985) Steady growth cone currents revealed by a novel circulary vibrating probe: A possible mechanism underlying neurite growth. J. Neurosci. Res. 13: 257-283.

Friel, D. D., and R. W. Tsien (1988) Effects of NGF on Ca channel distribution in PC-12 cells. Biophys. J. 53: 430a.

Fukuda, J., and M. Kameyama (1979) Enhancement of Ca spikes in nerve cells of adult mammals during neurite growth in tissue culture. Nature 279: 546-548.

Greene, L. A., and A. S. Tischler (1982) PC12 pheochromocytoma cultures in neurobiological research. Adv. Cell. Neurobiol. 3: 373414.

Grinvald, A., and I. C. Farber (1981) Optical recording of calcium action potentials from growth cones of cultured neurons with a laser microbeam. Science 212: 1164-1167.

Hamill, O. P., A. Marty, E. Neher, B. Sakmann, and F. J. Sigworth (1981) Improved patch-clamp techniques for high-resolution current recording from cells and cell-free membrane patches. Pfluegers Arch. 391: 85-100.

Hodgkin, A. L., and W. A. H. Rushton (1946) The electrical constants of a crustacean nerve fibre. Proc. R. Soc. London 133: 444-479.

Jacobs, J. R., and J. K. Stevens (1986) Changes in the organization of the neuritic cytoskeleton during nerve growth factor-activated differentiation of PC12 cells: A serial electron microscopic study of the development and control of neurite shape. J. Cell Biol. 103: 895-906.

Johnston, D., and T. H. Brown (1983) Interpretation of voltage-clamp measurements in hippocampal neurons. J. Neurophysiol. 50: 464486.

Kongsamut, S., and R. J. Miller (1986) Nerve growth factor modulates the drug sensitivity of neurotransmitter release from PC-12 cells. Proc. Natl. Acad. Sci. USA 83: 2243-2247.

Landis, S. C. (1983) Neuronal growth cones. Annu. Rev. Physiol. 45: $567-580$.

Lipscombe, D., D. V. Madison, M. Poenie, H. Reuter, R. Y. Tsien, and R. W. Tsien (1988) Spatial distribution of calcium channels and cytosolic calcium transients in growth cones and cell bodies of sympathetic neurons. Proc. Natl. Acad. Sci. USA 85: 2398-2402.

Llinás, R. R. (1979) In The Neurosciences: Fourth Study Program, F. O. Schmitt and F. G. Wordon, eds., pp. 555-571, MIT Press, Cambridge, MA.

Llinás, R., and M. Sugimori (1980) Electrophysiological properties of in vitro Purkinje cell dendrites in mammalian cerebellar slices. J. Physiol. (Lond.) 305: 197-213.

Llinás, R., and Y. Yarom (1981) Electrophysiology of mammalian inferior olivary neurons in vitro. Different types of voltage-dependent ionic conductances. J. Physiol. (Lond.) 315: 549-567.

Lux, H. D., and A. M. Brown (1984) Patch and whole cell calcium currents recorded simultaneously in snail neurons. J. Gen. Physiol. 83: 727-750.

Marom, S., and D. Dagan (1987) Calcium current in growth balls from isolated Helix aspersa neuronal growth cones. Pfluegers Arch. 409: 578-581.

Mattson, M. P., and S. B. Kater (1987) Calcium regulation of neurite elongation and growth cone motility. J. Neurosci. 7: 4034-4043.
Meiri, H., M. E. Spira, and I. Parnas (1981) Membrane conductance and acton potential of a regenerating axonal tip. Science 211: 709712 .

Miller, R. J. (1987) Multiple calcium channels and neuronal function. Science 235: 46-52.

Niesen, C. E., T. J. Blaxter, and P. L. Carlen (1987) Electrophysiological, pharmacological and spatial separation of three calcium currents in rat dentate granule neurons. Soc. Neurosci. Abstr. 13: 103.

Nowycky, M. C., A. P. Fox, and R. W. Tsien (1985) Three types of neuronal calcium channel with different agonist sensitivity. Nature 316: 440-443.

O'Lague, P. H., S. L. Huttner, C. A. Vandenberg, K. Morrison-Graham, and R. Horn (1985) Morphological properties and membrane channels of the growth cones induced in PC12 cells by nerve growth factor. J. Neurosci. Res. 13: 301-321.

Plummer, M. R., D. E. Logothetis, and P. Hess (1989) Elementary properties and pharmacological sensitivities of calcium channels in mammalian peripheral neurons. Neuron 2 : 1453-1463.

Rall, W. (1977) Cellular biology of neurons. In Handbook of Physiology. The Nervous System, Vol. 1, J. M. Brookhart and V. B. Mountcastle, eds., pp. 39-97, Am. Phys. Soc., Bethesda, MD.

Ross, W. N., H. Aréchiga, and J. G. Nicholls (1988a) Optical recording of calcium and voltage transients following impulses in cell bodies and processes of identified leech neurons in culture. J. Neurosci. 7: 3877-3887.

Ross, W. N., H. Aréchiga, and J. G. Nicholls (1988b) Influence of substrate on the distribution of calcium channels in identified leechneurons in culture. Proc. Natl. Acad. Sci. USA 85: 4075-4078.

Schubert, D., M. LaCorbiere, C. Whitlock, and W. Stallcup (1978) Alterations in the surface properties of cells responsive to nerve growth factor. Nature 273: 718-723.

Small, R. K., and K. H. Pfenninger (1984) Components of the plasma membrane of growing axons. I. Size and distribution of intramembrane particles. J. Cell Biol. 98: 1422-1433.

Srinivasan, Y., L. Elmer, J. Davis, V. Bennet, and K. Angelides (1988) Ankyrin and spectrin associate with voltage-dependent sodium channels in brain. Nature 333: 177-180.

Streit, J., and H. D. Lux (1986) Voltage-dependent calcium currents in PC-12-growth cones and cells: Relation to NGF-induced neurite growth. Pfluegers Arch. 406: R27.

Streit, J., and H. D. Lux (1987) Voltage dependent calcium currents in PCl 2 growth cones and cells during NGF-induced cell growth. Pfluegers Arch. 408: 634-641.

Streit, J., and H. D. Lux (1988) Calcium current distribution in PC12 cells during NGF-induced differentiation. Pfluegers Arch. 411: R143.

Tsien, R. Y., and T. J. Rink (1980) Neutral carrier ion selective microelectrodes for measurement of intracellular free calcium. Biochem. Biophys. Acta 599: 623-638.

Willard, A. L. (1980) Electrical excitability of outgrowing neurites of embryonic neurons in cultures of dissociated neural plate of Xenopus laevis. J. Physiol. (Lond.) 301: 115-128.

Yaari, Y., B. Hamon, and H. D. Lux (1987) Development of two types of calcium channels in cultured mammalian hippocampal neurons. Science 235: 680-682. 